\title{
Storage-retrieval analysis of acoustic similarity
}

\author{
RICHARD CHECHILE \\ Tufts University, Medford, Massachusetts 02155
}

\begin{abstract}
A statistical procedure that separates storage from retrieval was used to study the acoustic similarity effect as a function of retention interval in the Brown-Peterson paradigm. Both storage and retrieval components showed reliable and independent changes with retention interval, but only the storage component was affected by acoustic similarity. Hence, acoustic similarity affects trace durability and retrieval plays no essential role in the similarity effect. This finding is inconsistent with the address hypothesis (cf. Baddeley, 1968). It is argued that acoustic similarity induces subjects to encode the target item in a confused fashion, particularly in regard to order information.
\end{abstract}

A phenomenon of continued interest in short-term memory research is the acoustic or articulatory similarity effect. The phenomenon is basically that phonemically similar material is more susceptible to error than phonemically dissimilar material, particularly at shorter retention intervals (Conrad, 1967; Peterson \& Johnson, 1971; Tell, 1972). Despite considerable work on this effect, there is still some confusion as to the detailed mechanisms that underlie the phenomenon. For example, Murray (1968) has argued that the similarity effect is due to storage processes, whereas Baddeley (1968) argues that it is due to retrieval processes.

Using a serial probe task, Murray (1968) found acoustic confusions if the target material was presented either aurally or visually under standard learning conditions. However, if the subjects were required to suppress articulation of the target information by vocalizing a neutral word (e.g., "the"), then the similarity effect disappeared for visually presented material but persisted for aurally presented material. This finding has been replicated by Peterson and Johnson (1971), and these experiments suggest that a phonemic storage is necessary for the similarity effect to occur. Given, however, a phonemic representation of the target information, does acoustic similarity have its effect on the availability of the target information or is the information available but difficult to retrieve at the time of test? The experiments by Murray (1968) and Peterson and Johnson (1971) do not resolve that issue.

Baddeley (1968) argued that the similarity effect cannot be due to input and storage processes because he supposedly found identical decay rates between acoustically similar and dissimilar material. Baddeley reasons that the acoustic similarity effect is due to retrieval

This research was supported in part by a grant from the National Institute of Mental Health, MH-12672-06. Requests for reprints should be sent to Dr. Richard Chechile, Department of Psychology, Tufts University, Medford, Massachusetts 02155. The author would like to acknowledge the able assistance of Jeffrey Lawrence for his hard work in running subjects and scoring the data. processes, since the phenomenon could not be due to storage. The implicit assumption, however, is that the rate of change in correct recall in the Brown-Peterson paradigm is a measure of storage alone, but, clearly, this assumption has not been convincingly demonstrated. Moreover, in the first two of Baddeley's experiments, there were large differences in immediate recall between the acoustically similar and dissimilar conditions which produced difficulties in establishing decay rates. If a percentage deviation from immediate recall was computed with the Baddeley data, there would be a tendency for a faster rate of forgetting in the acoustically similar condition. Additionally, in the third experiment reported by Baddeley, an immediate recall standardization procedure used a different number of items in the two conditions to bring the immediate recall for both conditions to about $75 \%$. As Shulman (1971) has pointed out, the differing amount of target information in the Baddeley experiment makes impossible the interpretation of changes in correct recall with retention interval. Consequently, it seems that the Baddeley experiments have not convincingly ruled out the role of storage processes in the acoustic similarity effect. Moreover, the mechanism for the effect remains an open question, since there was no direct evidence for retrieval as the mechanism of the similarity effect in those experiments.

Theoretically, there are several plausible mechanisms for the phonemic similarity effect. One explanation that interprets the acoustic similarity effect as a storage phenomenon is the hypothesis that intraitem similarity affects the ease of encoding the target information in memory. Acoustically similar items are generally high in articulatory similarity and articulatory confusions as well (Wickelgren, 1965a, 1966), so these items may be more difficult to encode and store. A second hypothesis that stresses retrieval processes could be Baddeley's (1968) address hypothesis. According to this hypothesis, acoustically similar material creates interference at the time of test in the subjects' decision processes as to which unit to output. Thus, in the second hypothesis, all 
of the target information is available in memory but is not retrievable at the time of test. For example, if the subject was given the letter string BTD, then, according to the address hypothesis, the subject would have available in memory the individual letters but have difficulty in deciding the output order of the letters. Consequently, the major purpose of the present study is to clarify the mechanisms of acoustic similarity in terms of storage and retrieval processes.

Recently a procedure has been developed (e.g., Chechile, 1973; Chechile \& Meyer, 1976) that enables the separation of storage and retrieval effects in memory retention. A necessary condition for this procedure is the random supplementing of the usual recall trials with old and distractor recognition trials. Also, on recognition trials 3-point confidence judgments are required (where a 3 rating denotes highest confidence and no guessing). With the general task, Chechile \& Meyer (1976) develop several probabilistic task analyses of the recall, old, and distractor recognition trials, the most general being their Model 4. An extensive discussion of this model, along with the mathematical details for estimating storage and retrieval on an individual subject basis, is given in Chechile and Meyer (1976). However, a briefer verbal presentation of the model will be given here.

Storage, $\theta_{\mathbf{s}}$, is defined as the proportion of times the target information has been sufficiently stored, whereas retrieval, $\theta_{\mathrm{r}}$, is defined as the proportion of times there is sufficient storage for the subject to successfully retrieve the memory trace. Notice that storage is defined as a probability of representation at the time of test. Thus, $\theta_{\mathrm{s}}$ corresponds to the success rate for trace encoding and maintenance, while $\theta_{\mathrm{I}}$ corresponds to the success rate for retrieval at the time of test, given that the target is stored. Obviously, the proportion correctly recalled is just $\theta_{\mathrm{s}} \theta_{\mathrm{r}}$, since a correct production of a complex target item, that is unlikely to be guessed, requires both storage and retrieval.

The model for old recognition is shown as a tree diagram in Figure 1. It is assumed that when the subject has sufficiently stored the target, he will give a "yes 3 " response. Essentially this assumption implies that there are no retrieval difficulties in old recognition when there is sufficient storage. However, with insufficient storage the subject may guess "yes" correctly with probability $\theta_{\mathrm{g}}$. The parameters $\theta_{1}, \theta_{2}, \theta_{3}$, and $\theta_{4}$ correspond to the rating processes as illustrated in Figure 1.

The model for distractor recognition is shown in Figure 2. It is assumed that when there is sufficient storage the distractor item elicits the target encoding with probability $\theta_{\mathrm{dr}}$. If there is both sufficient storage and successful distractor retrieval, the subject responds "no 3." Otherwise, the subject guesses and rates as illustrated in Figure 2.

It should be pointed out that the same storage parameter appears on recall, old, and distractor recognition trials. This assumption should be reasonable because the randomization procedure used insures that subjects have no clue prior to testing as to the type of test procedure used on any particular trial.

Previous research has provided validating evidence for the separation process. One important source of validation data is the empirically determined statistical independence between $\theta_{\mathrm{s}}$ and $\theta_{\mathrm{r}}$ (e.g., Butler \& Chechile, 1976; Chechile, 1973; Chechile \& Butler, 1975; Chechile \& Meyer, 1976; Gerrein, 1976; Skoff \& Chechile, 1977; Chechile \& Gordon, Note 1 ; Gerrein \& Chechile, Note 2). This finding clearly shows that the separation technique is successful in statistically teasing apart separate components of recall performance and also shows that the measures of storage and retrieval are uncontaminated. Another important source of validating data is that providing the subject with more time to search memory

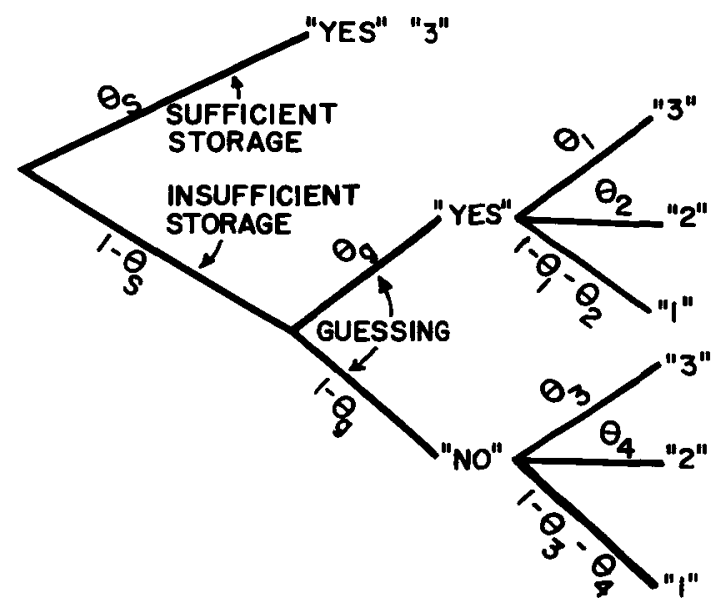

Figure 1. Tree diagram for the events in old recognition.

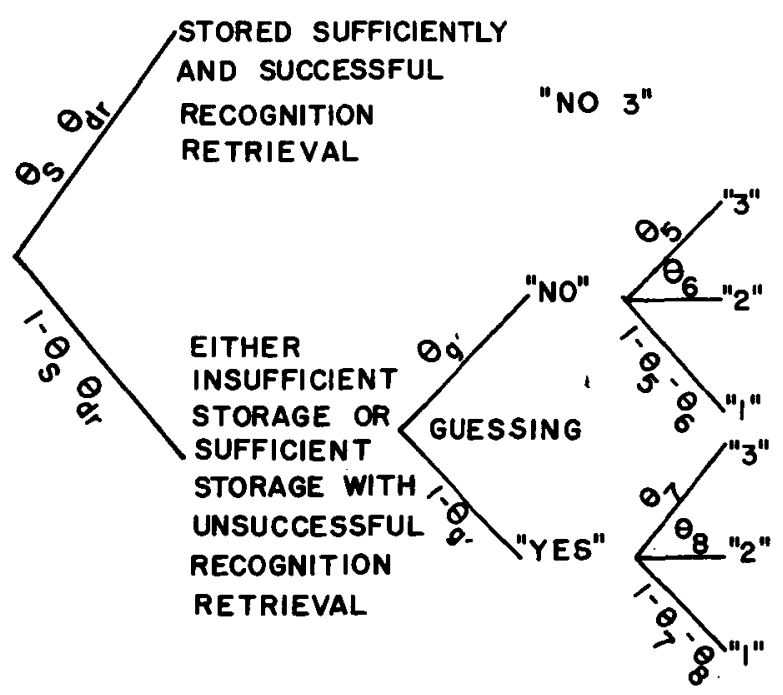

Figure 2. Tree diagram for the events in distractor recognition. 
definitely improves recall performance, but only the retrieval component, $\theta_{\Sigma}$, improves with this manipulation (Chechile \& Meyer, 1976). Clearly, this is the only result that should have occurred if the separation procedure is valid. Thus the separation technique is a powerful information processing tool to examine and, hopefully, resolve the issue concerning the difficulty produced by acoustic similarity.

\section{METHOD}

\section{Subjects}

Fifty undergraduates at Tufts University volunteered to participate in the experiment. All had normal hearing and spoke English as their native language.

\section{Materials}

The stimulus material for this experiment consisted of 240 consonant trigrams selected from Witmer (1935) and ranging in meaningfulness from $13 \%$ to $50 \%$. The acoustically similar trigrams were arranged so that the three letters shared a common phoneme. The similar trigrams were selected from either the F, L, M, N, S or the B, C, D, G, P, T, V, Z letter pools (e.g., PGT). The acoustically dissimilar trigrams, however, were constructed so that no internationally defined phoneme, according to Fairbanks (1960), was common among any of the letters. The dissimilar trigrams were selected from various pools made up of combinations of the above two groups and including the dissimilar letters $H, K, R$ (e.g., RZM). Thus, in the similar condition a total pool of 13 letters were used and it was necessary to use 16 letters in the dissimilar pool. Finally, the distractor recognition items themselves were either similar or dissimilar, depending on the experimental condition; however, no distractor item ever shared any common letters with the target item with which it was paired. Distractor items in the similar condition came from either the same letter pool as the target or from the other letter pool.

\section{Design}

The subjects were randomly assigned to either the acoustically similar (AS) or the acoustically dissimilar (AD) condition, but each subject was tested at the 0-, 3-, and 9-sec retention intervals. Of the 30 trials, 10 were recall trials, 10 were old recognition trials, and 10 were distractor recognition trials. Both type of test trial and retention interval were randomized as to the order of occurrence among the $\mathbf{9 0}$ trials. The random intermixing of recall, old, and distractor recognition trials is required in order to execute the storage-retrieval separation procedure.

\section{Apparatus and Procedure}

The tape-recorded instructions and experimental trials were played on a Sony TC -630 stereo tape recorder and delivered over Koss Pro-4A headphones. Each trial consisted of three stages: the presentation of the target trigram, an interpolated interval, and a test cue. A high-pitched ready tone preceded the presentation of the target trigram by $1 / 2 \mathrm{sec}$. The target trigrams were read in a female voice at a rate of 1 letter per $1 / 2 \mathrm{sec}$. Immediately after the target item presentation, a randomly selected four-digit number was presented in a male voice. The subject was required to repeat the trigram and the number and begin counting backward by threes from the four-digit number. After the 0,3 , or $9 \mathrm{sec}$ of interpolated activity, one of two types of test cues was spoken in the female voice. If the cue was the word "recall," the subject was allowed $3 \mathrm{sec}$ to report the target trigram. If however, a trigram was presented as a test cue, the subjects had to identify whether or not the test cue was the same as the target trigram by a yes or no response. Following the recognition response, the subjects were instructed to give a confidence rating for their response. The subjects were instructed to use the " 3 " rating if they were absolutely sure, the " 2 " rating if they gave an "educated" guess based on partial information, and the " 1 " rating if they just randomly guessed. The subjects were not informed as to which type of test cue would be used before the test. Following each response period, the subjects were given a $2 \frac{1}{2}$-sec intertrial rest interval. Finally, each subject received four practice trials in the same fashion as the previously described trials before the beginning of the experimental session.

\section{RESULTS}

The mean proportions for the various response categories are shown in Table 1. It is apparent that both proportions correctly recalled and recognized declined rapidly with increasing retention interval. The recall scores in Table 1 represent an order scoring, where the subjects are required to produce all of the target letters in the correct order. If a liberal item scoring is used, which disregards incorrect letter orders, then the pro. portions correctly recalled in the 0-, 3-, and 9-sec conditions are, respectively, $.976, .636$, and .268 in the $\mathrm{AD}$ situation and $.971, .604$, and .221 in the AS condition. Combining across the three retention intervals, there is lower correct recall for order scoring in the acoustically similar conditions $[t(48)=2.98, p<.01]$, but not when using the liberal item scoring $[\mathrm{t}(48)=1.55, \mathrm{p}>.1]$.

Table 1

Mean Proportion for Each Response Category

\begin{tabular}{|c|c|c|c|c|c|c|c|c|c|c|c|c|c|}
\hline \multirow{3}{*}{$\begin{array}{c}\text { Retention } \\
\text { Interval } \\
\text { (sec) }\end{array}$} & \multirow{3}{*}{$\begin{array}{l}\text { Cor- } \\
\text { rect } \\
\text { Recall }\end{array}$} & \multicolumn{6}{|c|}{ Old Recognition } & \multicolumn{6}{|c|}{ Distractor Recognition } \\
\hline & & \multicolumn{3}{|c|}{ Yes } & \multicolumn{3}{|c|}{ No } & \multicolumn{3}{|c|}{ Yes } & \multicolumn{3}{|c|}{ No } \\
\hline & & 1 & 2 & 3 & 1 & 2 & 3 & 1 & 2 & 3 & 1 & 2 & 3 \\
\hline \multicolumn{14}{|c|}{ Acoustically Similar } \\
\hline $\begin{array}{l}0 \\
3 \\
9\end{array}$ & $\begin{array}{l}.896 \\
.476 \\
.184\end{array}$ & $\begin{array}{l}.008 \\
.000 \\
.028\end{array}$ & $\begin{array}{l}.008 \\
.116 \\
.332\end{array}$ & $\begin{array}{l}.956 \\
.800 \\
.392\end{array}$ & $\begin{array}{l}.000 \\
.000 \\
.060\end{array}$ & $\begin{array}{l}.016 \\
.044 \\
.132\end{array}$ & $\begin{array}{l}.012 \\
.040 \\
.056\end{array}$ & $\begin{array}{l}.000 \\
.000 \\
.016\end{array}$ & $\begin{array}{l}.000 \\
.028 \\
.028\end{array}$ & $\begin{array}{l}.000 \\
.016 \\
.004\end{array}$ & $\begin{array}{l}.004 \\
.020 \\
.072\end{array}$ & $\begin{array}{l}.004 \\
.140 \\
.140\end{array}$ & $\begin{array}{l}.992 \\
.804 \\
.740\end{array}$ \\
\hline \multicolumn{14}{|c|}{ Acoustically Dissimilar } \\
\hline $\begin{array}{l}0 \\
3 \\
9\end{array}$ & $\begin{array}{l}.976 \\
.604 \\
.256\end{array}$ & $\begin{array}{l}.004 \\
.000 \\
.040\end{array}$ & $\begin{array}{l}.004 \\
.012 \\
.172\end{array}$ & $\begin{array}{l}.984 \\
.956 \\
.596\end{array}$ & $\begin{array}{l}.000 \\
.000 \\
.024\end{array}$ & $\begin{array}{l}.004 \\
.024 \\
.112\end{array}$ & $\begin{array}{l}.004 \\
.008 \\
.056\end{array}$ & $\begin{array}{l}.000 \\
.000 \\
.012\end{array}$ & $\begin{array}{l}.000 \\
.016 \\
.036\end{array}$ & $\begin{array}{l}.004 \\
.008 \\
.012\end{array}$ & $\begin{array}{l}.016 \\
.008 \\
.052\end{array}$ & $\begin{array}{l}.028 \\
.040 \\
.220\end{array}$ & $\begin{array}{l}.952 \\
.928 \\
.668\end{array}$ \\
\hline
\end{tabular}


Table 2

Group Mean and Standard Error for Storage, $\theta_{g}$, Retrieval, $\theta_{\mathbf{r}}$, Old Recognition Guessing, $\theta_{g}$, and Distractor Recognition Guessing, $\theta_{\mathbf{g}}$

\begin{tabular}{|c|c|c|c|c|c|c|c|c|c|c|}
\hline \multirow[t]{2}{*}{. } & \multicolumn{2}{|c|}{$0 \mathrm{sec}$} & \multicolumn{4}{|c|}{$3 \sec$} & \multicolumn{4}{|c|}{$9 \mathrm{sec}$} \\
\hline & $\theta_{\mathbf{s}_{\mathbf{0}}}$ & ${ }^{\theta} \mathbf{r}_{0}$ & $\theta_{\mathbf{s}}$ & $\theta_{\mathbf{r}}$ & $\theta_{g}$ & $\theta_{\mathbf{g}^{\prime}}$ & $\theta_{\mathbf{s}}$ & $\theta_{\mathbf{r}}$ & $\theta_{\mathbf{g}}$ & $\theta_{\mathbf{g}^{\prime}}$ \\
\hline & \multicolumn{10}{|c|}{ Acoustically Similar } \\
\hline \multirow[t]{2}{*}{$\begin{array}{l}\text { Mean } \\
\text { Standard Error }\end{array}$} & $\begin{array}{l}.896 \\
.020\end{array}$ & $\begin{array}{l}1 \\
0\end{array}$ & $\begin{array}{l}.832 \\
.032\end{array}$ & $\begin{array}{l}.681 \\
.044\end{array}$ & $\begin{array}{l}.648 \\
.075\end{array}$ & $\begin{array}{l}.887 \\
.048\end{array}$ & $\begin{array}{l}.461 \\
.049\end{array}$ & $\begin{array}{l}.553 \\
.072\end{array}$ & $\begin{array}{l}.634 \\
.052\end{array}$ & $\begin{array}{l}.924 \\
.022\end{array}$ \\
\hline & \multicolumn{10}{|c|}{ Acoustically Dissimilar } \\
\hline $\begin{array}{l}\text { Mean } \\
\text { Standard Error }\end{array}$ & $\begin{array}{l}.972 \\
.009\end{array}$ & $\begin{array}{l}1 \\
0\end{array}$ & $\begin{array}{l}.966 \\
.013 \\
\end{array}$ & $\begin{array}{l}.732 \\
.047\end{array}$ & $\begin{array}{l}.773 \\
.080\end{array}$ & $\begin{array}{l}.884 \\
.059\end{array}$ & $\begin{array}{l}.592 \\
.051 \\
\end{array}$ & $\begin{array}{l}.494 \\
.062\end{array}$ & $\begin{array}{l}.598 \\
.072\end{array}$ & $\begin{array}{l}.872 \\
.042\end{array}$ \\
\hline
\end{tabular}

However, the acoustic similarity effect is demonstrated with the recognition measures [i.e., the combined false alarms and misses across the three retention intervals are higher in the acoustically similar condition, $\mathrm{t}(48)=2.17$, $\mathrm{p}<.05]$. Further statistical tests for these data will not be presented here since these data have not been corrected for recognition guessing and rating factors. In essence, those factors are taken into account in the storageretrieval separation analysis. The resulting model estimates of storage $\left(\theta_{\mathrm{S}}\right)$, retrieval $\left(\theta_{\mathrm{r}}\right)$, old recognition guessing when there is insufficient storage $\left(\theta_{\mathrm{g}}\right)$, and distractor regognition guessing when there is insufficient storage $\left(\theta_{\mathrm{g}}\right)$ were determined by the modes of the distributions shown in Equations 16-24 from Chechile and Meyer (1976). These parameter estimates were determined for each subject at each non-0-sec retention interval and were based on both the recall and recognition data. Due to the number of parameters in the model, the model completely specifies the data, so there is a perfect goodness-of-fit.

For the immediate recall conditions it is assumed that the proportion correctly recalled is simply a measure of immediate storage, $\theta_{s_{\mathrm{O}}}$. Errors in immediate recall are thus considered to be misperceptions and constitute a failure to store sufficiently the appropriate memory trace. Consequently, immediate retrieval, $\theta_{\mathbf{r}_{0}}$, is assumed to be one. The resulting group means and standard errors of the means for storage, retrieval, and guessing measures are displayed in Table 2.

There are significant differences in the storage measure between the acoustic similarity conditions, both at the $3-\sec [t(48)=3.88, p<.0002]$ and at the $9-\sec$ retention intervals $[\mathrm{t}(48)=1.85, \mathrm{p}<.05]$, although the magnitude of the similarity effect for storage tends to be reduced for the longer retention interval. This finding for storage is consistent with the Tell (1972) findings on proportion correctly recalled. Moreover, the retrieval measure is not significantly different at either retention interval [largest $\mathrm{t}(48)=.79, \mathrm{p}>.2$ ] .

The ostensible difference in storage (at both the 3 and 9-sec intervals) as a function of acoustic similarity, however, needs more careful examination, since initial storage for the acoustically similar condition is lower $[t(48)=3.46, p<.001]$. In order to correct for these different baselines, the proportion deviation from initial storage and retrieval scores was computed for each subect [i.e., $\mathrm{P}_{\mathrm{S}}=\left(\theta_{\mathrm{s}_{\mathrm{O}}}-\theta_{\mathrm{S}}\right) / \theta_{\mathrm{s}_{\mathrm{o}}}$ and $\mathrm{P}_{\mathrm{r}}=\left(\theta_{\mathrm{r}_{\mathrm{o}}}-\theta_{\mathrm{r}}\right) / \theta_{\mathrm{r}_{\mathrm{o}}}$ ]. It can be easily demonstrated that the overall deviation from initial correct recall, $P_{F}$, is $P_{S}+P_{r}-P_{s} \cdot P_{r}$ (cf. Chechile \& Meyer, 1976, p. 271). Notice, for the present data, that the $P_{s}$ transformation will tend to reduce the size of the 3- and 9-sec storage differences between the acoustical similarity conditions. The means for the $P_{F}$, $P_{\mathbf{s}}$, and $P_{\mathbf{r}}$ measures are displayed in Figure 3 in Panels 1 , 2 , and 3 , respectively.

With the exception of the storage measure at the 3-sec retention interval, an analysis of the data displayed in Figure 3 indicates a highly significant decrement from immediate baseline for each measure [i.e., smallest $t(24)=5.262, p<.00002]$. Furthermore, despite the fact that both storage and retrieval show increased failures by the $9-\mathrm{sec}$ retention interval, the correlation coefficient across subjects between the proportion storage change and the proportion retrieval change is .008 . Also, a scatter plot of the data did not show a nonlinear relationship between the storage and retrieval measures that might have been missed in the correlational analysis. Hence, these data again show the

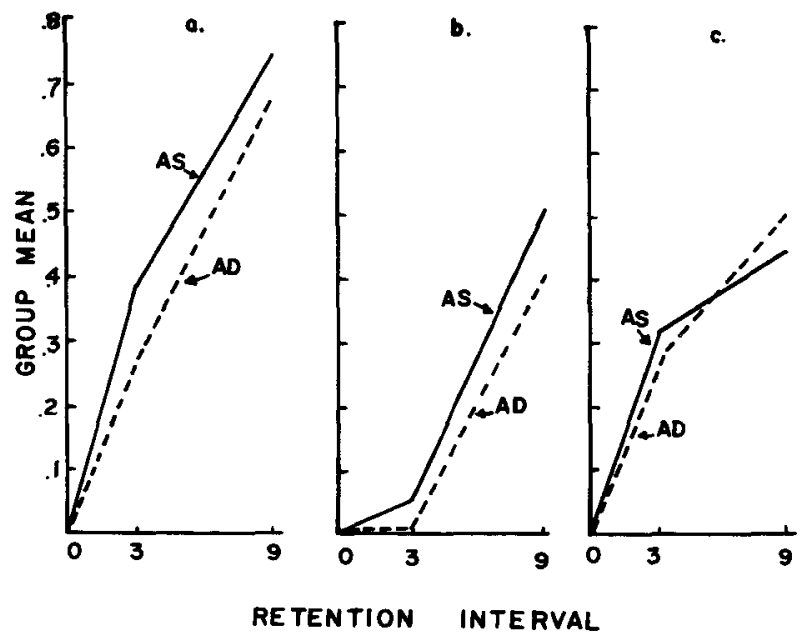

Figure 3. The retention function for percent deviation from 0-ece performance for (1) correct recall, (2) the storage probability, and (3) the retrieval probability. 
independence between the storage and retrieval measures found in previous studies (e.g., Butler \& Chechile, 1976; Chechile, 1973; Chechile \& Butler, 1975; Chechile \& Meyer, 1976; Gerrein, 1976; Skoff \& Chechile, 1977; Chechile \& Gordon, Note 1 ; Gerrein \& Chechile, Note 2). Consequently, the storage and retrieval measures of the present study show evidence of internal validation.

Combining across retention intervals, there is a tendency for greater combined $\mathrm{P}_{\mathrm{F}}$ scores in the acoustically similar condition $[t(48)=1.66, p<.06]$. Furthermore, the storage measure accounts for $95 \%$ of the effect produced by acoustic similarity in the overall $P_{F}$ measure. Thus, the effect of acoustic similarity found in the present study is attributable to storage differences, although the strength of the similarity effect for delayed testing is greatly reduced by the transformation to proportion deviation from 0 -sec performance.

An analysis of the guessing data, shown in Table 2, indicates no significant differences in either guessing measure, when combined across retention intervals, as a function of acoustic similarity. Furthermore, there are no significant differences between 3- and 9-sec retention intervals for either guessing measure when combined across all subjects. Combining across retention intervals, the distractor recognition guessing measure, $\theta_{\mathrm{g}}{ }^{\prime}$, is significantly greater than the old recognition guessing measure, $\theta_{\mathrm{g}}$, for both the AS condition $[\mathrm{t}(24)=3.55$, $\mathrm{p}<.001]$ and the $\mathrm{AD}$ condition $[\mathrm{t}(24)=2.53, \mathrm{p}<.01]$. This result is reasonable: It should be easier, based on fractional but insufficient information, to reject a false item than to accept a correct item, since only one mismatch is sufficient to reject a false item.

The parameters $\theta_{1} \ldots \theta_{8}$ are not computed since they are nuisance parameters associated with the rating process in recognition and are of little psychological interest. The distractor recognition retrieval parameter, $\theta_{\mathrm{dr}}$, is also a nuisance parameter of moderate interest. The mean values of $\theta_{\mathrm{dr}}$ in the 3- and 9-sec conditions are, respectively, .934 and .888 in the AS condition and .931 and .748 in the $\mathrm{AD}$ condition. There are no significant differences between the similarity conditions in terms of $\theta_{\mathrm{dr}}$.

Finally, the above statistical analyses were also reexamined with more advanced Bayesian inferential procedures (cf. Chechile \& Meyer, 1976, p. 285). All of the conclusions reached with the more familiar classical tests were also found with these Bayesian analyses. Consequently, the conclusions of the experimenter are robust with respect to the inferential model.

\section{DISCUSSION}

There are at least two important findings in this experiment. The first is that short-term memory failures that increase with retention interval are due to both storage and retrieval difficulties, but these factors are uncorrelated. The second finding is that the confusions due to phonemic similarity are due to storage difficulties alone.

Considering first the retention interval changes, it is particularly noteworthy that both storage and retrieval show reliable and uncorrelated changes. This result is a replication of retention interval effects reported in Chechile (1973), Chechile and Meyer (1976), Gerrein (1976), and Gerrein and Chechile (Note 2). Also, these studies have typically found that retention curves differ for the storage and retrieval measure. Plotted in terms of deviations from initial processing, the retrieval function is typically a concave-downward curve, whereas the storage function is concave upward. This difference in the shape of the retention functions lends further support to the independent nature of the storage and retrieval measures. Additional support for the independence is found in the fact that acoustic similarity affected only storage; if the storage and retrieval measures were confounded, acoustic similarity would most likely have affected both measures.

The conclusion that acoustic similarity affects only storage can be given even further support and clarification by an examination of the item and order information. In recall, the acoustic similarity effect is found only with the strict order scoring and is not found with liberal item scoring. This finding replicates Wickelgren (1965b). Recognition errors, however, were greater in the acoustically similar condition. This finding is interesting, considering that recognition could be based on item information alone, since the distractor probes did not share any letters in common with the target. Thus, recognition does nor require order information, as does the strict recall scoring. However, the greater number of recognition errors in the acoustically similar condition indicates that the actual recognition performance must have been based on both item and some order information. Presumably, in the acoustically similar condition, subjects tend to have more difficulty encoding acoustically similar items. The subjects tend to encode the letters in the wrong order and the confused encoding order induces the subjects to make recognition errors and strict-scoring recall errors. The increased number of recognition errors also clearly indicates that Baddeley's (1968) address hypothesis is incorrect. For example, on old recognition the subjects in the acoustically similar condition actually have a greater tendency to reject the correct order of the letters (presumably because it does not match with the confused encoded order). Hence, the confusion of order information is not an output interference effect, as suggested by Baddeley, but rather it is a consequence of a confused encoding of the order information. Moreover, the present storage-retrieval analysis indicates that, if a subject can overcome the difficulties of storing sufficiently a highly similar item, then the resulting retrieval for that item is virtually the same as for sufficiently stored dissimilar items.

Finally, the present study is somewhat relevant to the 
"acid-bath" theory of Posner and Konick (1966). In this theory, similarity corresponds to the concentration of the acid. Thus, similarity increases the rate of memory decay. Recently, Butler and Chechile (1976) have criticized the acid-bath theory in regard to proactive-interference effects in the Brown-Peterson task. In the present experiment, it also seems that the acid-bath theory cannot explain the effects of intraitem similarity on the decay rate. Using either the proportion correctly recalled or $\theta_{\mathrm{s}}$ or $\theta_{\mathrm{r}}$, there are no differences in the rate of decay between the similarity conditions. However, if a percent deviation from 0-sec performance is used, the high-similarity condition does increase the decay rate for $P_{F}$ and $P_{\mathbf{s}}$. The reason for the discrepancy between these two methods of establishing decay rates is the difference between the similarity groups at the 0 -sec retention interval. This result is interesting since the acid-bath theory cannot account for a difference at the 0-sec interval; yet, without that difference there would be no difference in decay rates even with percent deviation measures. Consequently, the current similarity data add further doubt of the validity of the acid-bath theory.

\section{REFERENCE NOTES}

1. Chechile, R. A., \& Gordon, T. Storage-retrieval analysis of paired-associate acquisition. Paper presented at the 84th annual meeting of the American Psychological Association, Washington, D. C., September 1976.

2. Gerrein, J. R., \& Chechile, R. Effect of organization on storage and retrieval processes in short-term memory. Paper presented at the 84th annual meeting of the American Psychological Association, Washington, D. C., September 1976.

\section{REFERENCES}

BADDELeY, A. D. How does acoustic similarity influence shortterm memory? Quarterly Journal of Experimental Psychology, 1968, 20. 249-264.

Butler, K., \& Chechile, R. "Acid bath" effects on storage and retrieval PI. Bulletin of the Psychonomic Society, 1976, 8, 349-352.
CFECHILE, R. A. The relative storage and retrieval losses in short-term memory as a function of the similarity and amount of information processing in the interpolated task. Unpublished doctoral dissertation, University of Pittsburgh, 1973.

Chechile, R., \& Butler, K. Storage and retrieval changes that occur in the development and release of PI. Journal of Verbal Learning and Verbal Behavior, 1975, 14, 430-437.

Chechile, R., \& MeYer, D. L. A Bayesian procedure for separately estimating storage and retrieval components of forgetting. Journal of Mathematical Psychology, 1976, 13, 269-295.

ConRaD, R. Interference or decay over short retention intervals? Journal of Verbal Learning and Verbal Behavior, 1967, 6, $49-54$.

Fairbanxs, G. Voice and articulation. New York: Harper \& Row, 1960.

GERREIN, J. R. Organizational, storage, and retrieval factors in alcohol-induced forgetting. Unpublished doctoral dissertation, Tufts University, 1976.

Murray, D. J. Articulation and acoustic confusability in shortterm memory. Journal of Experimental Psychology, 1968, 4, $679,684$.

Peterson, L. R., \& Johnson, S. T. Some effects of minimizing articulation on short-term retention. Journal of Verbal Learning and Verbal Behavior, 1971, 10, 346-354.

Posner, M., \& Konick, A. On the role of interference in short-term retention. Journal of Experimental Psychology, 1966, 72, 221-231.

Shulman, H. G. Similarity effects in short-term memory. Psychological Bulletin, 1971, 75, 399-415.

Skoff, B., \& ChEChile, R. A. Storage and retrieval processes in the serial position effect. Bulletin of the Psychonomic Society, 1977, 9. 265-268.

TelL, P. M. The role of certain acoustic and semantic features at short and long retention intervals. Joumal of Verbal Learning and Verbal Behavior, 1972, 11, 455-464.

WICKElGREN, W. A. Distinctive features and errors in short-term memory for English vowels. Joumal of the Acoustical Society of America, 1965, 33, 583-588. (a)

WiCKELGREN, W. A. Short-term memory for phonemically similar lists. American Journal of Psychology, 1965, 78, 567-579. (b)

Wickelgren. W. A. Distinctive features and errors in STM for English consonants. Journal of the Acoustical Society of America, 1966, 39, 388-398.

WITMER, L. R. The association value of three-place consonant syllables. Journal of Genetic Psychology, 1935, 47, 337-359.

(Received for publication December 15, 1976; accepted June 24,1977 . 